\title{
Influence of Vibrations on Convective Instability of Reaction Fronts in Liquids
}

\author{
K. Allali ${ }^{1}$, F. Bikany ${ }^{1}$, A. Taik ${ }^{1 *}$ and V. Volpert ${ }^{2}$ \\ ${ }^{1}$ University Hassan II, UFR-MASI, Dept. of Maths, B.P. 146, FST-Mohammadia, Morocco \\ ${ }^{2}$ University Lyon1, Institute Camille Jordan, UMR 5208, 69100 Villeurbanne, France
}

\begin{abstract}
Propagation of polymerization fronts with liquid monomer and liquid polymer is considered and the influence of vibrations on critical conditions of convective instability is studied. The model includes the heat equation, the equation for the concentration and the Navier-Stokes equations considered under the Boussinesq approximation. Linear stability analysis of the problem is fulfilled, and the convective instability boundary is found depending on the amplitude and on the frequency of vibrations.
\end{abstract}

Key words: convective instability, frontal polymerization, vibrations

AMS subject classification: 34E05, 34E10, 35K57, 76V05, 76E15

\section{Introduction}

In this work we study the influence of vibrations on convective instability of reaction front in liquids, where both the reactant and the product are in the liquid phase. In the case where the monomer is liquid and the polymer is solid, the influence of vibrations was studied in [1]. Convective instability of polymerization fronts in the case when the reactant and the product are liquid without vibrations was studied in [3]. In order the study the influence of vibrations on the instability of a polymerization front, we impose a harmonic oscillation of frequency $\sigma$, and amplitude $\lambda$ in the vertical direction upon the system containing the liquid monomer and liquid polymer. This oscillation causes a periodic acceleration, $b$, perpendicular to the liquid-liquid interface. The time

\footnotetext{
${ }^{*}$ Corresponding author. E-mail: taik_ahmed@yahoo.fr
} 
dependence of the instantaneous acceleration acting on the fluids is then given by $g+b(t)$, where $b(t)=\lambda \sin (\sigma)$.

\section{Governing Equations}

We consider the effect of vibrations on propagation of polymerization fronts when both of reactant and product are liquids. Navier-Stokes equations are taken under the Boussinesq approximation. We consider the case where the propagation of the reaction front is at the opposite direction with respect to gravity. The process is described by the following dimensionless model:

$$
\begin{gathered}
\frac{\partial \theta}{\partial t}+\mathbf{v} \cdot \nabla \theta=\Delta \theta+W_{Z}(\theta) \phi(\alpha) \\
\frac{\partial \alpha}{\partial t}+\mathbf{v} \cdot \nabla \alpha=W_{Z}(\theta) \phi(\alpha) \\
\frac{\partial \mathbf{v}}{\partial t}+(\mathbf{v} \cdot \nabla) \mathbf{v}=-\nabla p+P \Delta \mathbf{v}+P R(1+\lambda \sin (\mu t))\left(\theta-\theta_{0}\right) \gamma \\
\nabla \cdot \mathbf{v}=0 .
\end{gathered}
$$

This system is considered in $3 D$ space. It is supplemented with the following conditions:

$$
(z \rightarrow+\infty, z \rightarrow-\infty), \quad \theta=(-1,0), \quad \alpha=(1,0) \text { and } \mathbf{v}=(0,0)
$$

We use the following notation:

$$
W_{Z}(\theta)=Z \exp \left(\frac{\theta}{Z^{-1}+\delta \theta}\right), \quad Z=\frac{q E}{R_{0} T_{b}^{2}}, \quad c^{2}=\frac{k_{0} \kappa}{q} \frac{R_{0} T_{b}^{2}}{E} \exp \left(\frac{-E}{R_{0} T_{b}}\right) .
$$

Here $\theta=\left(T-T_{b}\right) / q$ is the reduced temperature, $\alpha$ the depth of conversion, $\mathbf{v}=\left(v_{x}, v_{y}, v_{z}\right)$ the velocity of the medium, $p$ the pressure, the parameter $\theta_{0}$ is defined by $\theta_{0}=\left(T_{b}-T_{0}\right) / q$, $T_{0}$ is the mean value of the temperature $T, T_{b}$ is the burned temperature given by $T_{b}=T_{i}+q, T_{i}$ is the initial temperature, $q$ the adiabatic heat release. $E$ is the activation energy assumed to be large, $Z$ is the Zeldovich number, $R_{0}$ is the universal gas constant and $k_{0}$ the preexponential factor, $\delta$ is defined by $\delta=R_{0} T_{b} / E, R$ is the Rayleigh number and $P$ the Prandtl number that are defined by $R=g \beta q \kappa^{2} / \nu c_{1}^{3}$ and $P=\nu / \kappa, \nu$ the coefficient of kinematic viscosity, $\kappa$ the coefficient of thermal diffusivity, $g$ the gravity acceleration, $\mu=2 \kappa \sigma / c_{1}^{2}$ where $c_{1}=c / \sqrt{2}$, $c$ gives the velocity of the stationary front in the limit of large Zeldovich number [5], $\lambda$ and $\sigma$ are respectively the amplitude and the frequency of the vibrations. Here $\beta$ denotes the coefficient of thermal expansion, $\gamma$ is the unit vector in the vertical direction (upward). Finally, $\nabla$ and $\Delta$ denote the standard differential operators.

If the medium is at rest, that is $\mathbf{v}=0$, then there exists a stationary propagating front with the velocity and temperature distribution that can be found asymptotically for large values of the Zeldovich number $[1,3,4]$. In the case of the zero order reaction we have:

$$
(\theta(x, y, z, t), \alpha(x, y, z, t))=\left\{\begin{array}{ll}
(0,1) & \text { if } z<0, \\
\left.e^{-z}-1,0\right) & \text { if } z>0,
\end{array}, \phi(\alpha)= \begin{cases}1 & \text { if } \alpha<1 \\
0 & \text { if } \alpha=0\end{cases}\right.
$$




\section{Approximation of infinitely narrow reaction zone, interface problem}

To study analytically this problem we reduce it to a singular perturbation problem where the reaction zone is supposed to be infinitely narrow and the reaction term is neglected outside the reaction zone (Zeldovich- Frank Kamanetsky method [7]). To complete the problem some nonlinear jump conditions at the reaction zone are needed. This is a common approach for combustion problems $[2,3,4,6]$. We perform a formal asymptotic analysis with $\epsilon=1 / Z$ taken as a small parameter and we obtain the following closed interface problem for $z>\zeta$ (in the unburnt medium) and for $z<\zeta$ (in the burnt medium) where $\zeta(x, y, t)$ denotes the location of the reaction zone:

$$
\begin{gathered}
\frac{\partial \theta}{\partial t}+(\mathbf{v} \cdot \nabla) \theta=\Delta \theta, \quad \nabla \cdot \mathbf{v}=0, \\
\frac{\partial \mathbf{v}}{\partial t}+(\mathbf{v} . \nabla) \mathbf{v}=-\nabla p+P \Delta \mathbf{v}+Q(1+\lambda \sin \mu t)\left(\theta+\theta_{0}\right) \gamma, \quad Q=R P, \\
\alpha=\left\{\begin{array}{ll}
1 & \text { if } z>\zeta(\text { unreacted medium) } \\
0 & \text { if } z<\zeta \text { (reacted medium) }
\end{array} .\right.
\end{gathered}
$$

We finally complete this system by the following jump conditions at the interface $z=\zeta$ :

$$
\begin{gathered}
\left.\theta\right|_{\zeta-0}=\left.\theta\right|_{\zeta+0}, \quad A=\left(1+\left(\frac{\partial \zeta}{\partial x}\right)^{2}+\left(\frac{\partial \zeta}{\partial y}\right)^{2}\right)^{-1}, \\
\left.\frac{\partial \theta}{\partial z}\right|_{\zeta=-0}-\left.\frac{\partial \theta}{\partial z}\right|_{\zeta=+0}=A\left(\frac{\partial \zeta}{\partial t}+\left.\left(v_{x} \frac{\partial \zeta}{\partial x}+v_{y} \frac{\partial \zeta}{\partial y}-v_{z}\right)\right|_{\zeta=0}\right) \\
\left.\left(\frac{\partial \theta}{\partial z}\right)^{2}\right|_{\zeta=-0}-\left.\left(\frac{\partial \theta}{\partial z}\right)^{2}\right|_{\zeta=+0}=-\left.2 Z A \int_{-\infty}^{\theta}\right|_{\zeta=0} \exp \left(\frac{\tau}{Z^{-1}+\delta \tau}\right) d \tau \\
\left.v_{z}\right|_{\zeta=-0}=\left.v_{z}\right|_{\zeta=+0},\left.\frac{\partial v_{z}}{\partial z}\right|_{\zeta=-0}=\left.\frac{\partial v_{z}}{\partial z}\right|_{\zeta=+0},\left.\frac{\partial^{2} v_{z}}{\partial z^{2}}\right|_{\zeta=-0}=\left.\frac{\partial^{2} v_{z}}{\partial z^{2}}\right|_{\zeta=+0} \\
\left.\frac{\partial^{3} v_{z}}{\partial z^{3}}\right|_{\zeta=-0}-\left.\frac{\partial^{3} v_{z}}{\partial z^{3}}\right|_{\zeta=+0}=-\left.R A(A-1)(1+\lambda \sin (\mu t))\left(\frac{\partial \theta}{\partial z}\right)\right|_{\zeta=+0}
\end{gathered}
$$

The above free boundary problem is completed with the following conditions at infinity

$$
(z \rightarrow+\infty, z \rightarrow-\infty), \quad \theta=(-1,0) \text { and } \mathbf{v}=(0,0) .
$$

The closed problem (3.1)-(3.9) will be called the interface problem. In order to predict the influence of vibrations on the stability of the reaction front we have to solve this problem. To do so we use the asymtotic analysis theory in the next section. 


\section{Linear stability analysis}

In this section we perform the linear stability analysis of the steady-state solution for the interface problem (3.1)-(3.9), this problem has a travelling wave solution:

$$
\left(\theta_{s}(z-u t), \alpha_{s}(z-u t)\right)=\left\{\begin{array}{ll}
(0,1), & z_{2}<0, \\
\left(\exp \left(-u z_{2}\right)-1,0\right), & z_{2}>0,
\end{array}, z_{2}=z-u t,(u=c),\right.
$$

where $\mathrm{u}$ is the speed of the reaction front. This solution will be referred to as a basic solution. It is a stationary solution of (3.1)-(3.9) written in the moving coordinates. We seek the solution the following perturbed form: $\theta=\theta_{s}+\tilde{\theta}, p=p_{s}+\tilde{p}, v=v_{s}+\tilde{v}$ where $\tilde{\theta}, \tilde{p}$ and $\tilde{v}$ are respectively small perturbation of temperature, pressure and velocity. Here $\theta_{s}, p_{s}$ and $v_{s}$ are given by the basic solution. We substitute in (3.1)-(3.9), we obtain for the first-order terms:

$$
\begin{gathered}
\frac{\partial \tilde{\theta}}{\partial t}=\Delta \tilde{\theta}+u \frac{\partial \tilde{\theta}}{\partial z_{2}}- \begin{cases}\tilde{v}_{z} \theta_{s}^{\prime}, & z_{2}>\xi, \\
0, & z_{2}<\xi,\end{cases} \\
\frac{\partial \tilde{v}}{\partial t}=-\nabla \tilde{p}+P \Delta \tilde{v}+u \frac{\partial \tilde{\theta}}{\partial z_{2}}+Q(1+\lambda \sin (\mu t)) \tilde{\theta} \gamma, \quad z_{2} \neq \xi, \\
\operatorname{div}(\tilde{v})=0, \\
z_{2} \neq \xi,
\end{gathered}
$$

with $\xi=\zeta-u t$, let us denote $\left(\tilde{\theta}, \tilde{v}_{z}\right)=\left(\hat{\theta}_{1}, \hat{v}_{z 1}\right)$ for $z_{2}<\xi$ and $\left(\tilde{\theta}, \tilde{v}_{z}\right)=\left(\hat{\theta}_{2}, \hat{v}_{z 2}\right)$ for $z_{2}<\xi$, and consider the perturbations in the form:

$$
\left(\hat{\theta}_{i}, \hat{v}_{z i}, \xi\right)=\left(\theta_{i}\left(z_{2}, t\right) e^{\left(j\left(k_{1} x+k_{2} y\right)\right.}, v_{z i}\left(z_{2}, t\right) e^{\left(j\left(k_{1} x+k_{2} y\right)\right.}, \epsilon_{1}(t) e^{\left(j\left(k_{1} x+k_{2} y\right)\right.}\right),
$$

$k_{i}, i=1,2$ and $\epsilon_{1}$ are respectively the wave numbers (in $x$ and $y$ directions) and the amplitude of the perturbation, $j^{2}=-1$ and $w$ is the frequency. Taking into account that:

$$
\left.\theta\right|_{\xi= \pm 0}=\theta_{s}( \pm 0)+\xi \theta_{s}^{\prime}( \pm 0)+\tilde{\theta}( \pm 0),\left.\quad \frac{\partial \theta}{\partial z_{2}}\right|_{\xi= \pm 0}=\theta_{s}^{\prime}( \pm 0)+\xi \theta_{s}^{\prime \prime}( \pm 0)+\left.\frac{\partial \tilde{\theta}}{\partial z_{2}}\right|_{\xi= \pm 0},
$$

we linearize the jump conditions (3.5)-(3.9) and we substitute the equations (4.5) in the resulting linearization, Up to the higher order Terms we obtain the following relations:

$$
\begin{gathered}
\theta_{2}(0, t)-\theta_{1}(0, t)=u \epsilon_{1}(t), \quad \frac{\partial \theta_{2}}{\partial z}(0, t)-\frac{\partial \theta_{1}}{\partial z}(0, t)=-\epsilon_{1}(t) u^{2}-\frac{d \epsilon_{1}}{d t}(t)+v_{1}(0, t), \\
\epsilon_{1}(t) u^{2}+\frac{\partial \theta_{2}}{\partial z}(0, t)=-\frac{Z}{u} \theta_{1}(0, t), \quad \frac{\partial^{i} v_{2}}{\partial z^{i}}(0, t)=\frac{\partial^{i} v_{1}}{\partial z^{i}}(0, t) \quad i=0,1,2,3 .
\end{gathered}
$$

In order to find the perturbation terms we apply the transformation rot rot to equations (4.3). This allows us to eliminate the pressure and to consider only the velocity component $v_{z}$. Equations (4.3) become

$$
\frac{\partial}{\partial t} \Delta \tilde{v}_{z}-u \frac{\partial}{\partial z_{2}} \Delta \tilde{v}_{z}=P \Delta \Delta \tilde{v}_{z}+Q\left(1+\lambda \sin (\mu t)\left(\frac{\partial^{2}}{\partial x^{2}}+\frac{\partial^{2}}{\partial y^{2}}\right) \tilde{\theta}, z_{2} \neq \xi\right.
$$


Substituting (4.1), (4.5) into (4.2) and (4.8), we can have two systems according to $z_{2}<\xi$ and $z_{2}>\xi$ :

$$
\begin{aligned}
& w=v^{\prime \prime}-k^{2} v, \quad k=\sqrt{k_{1}^{2}+k_{2}^{2}}, \quad z_{2} \neq \xi, \\
& \frac{\partial w}{\partial t}-u w^{\prime}-P\left(w^{\prime \prime}-k^{2} w\right)=-Q k^{2}(1+\lambda \sin (\mu t)), \quad z_{2} \neq \xi \\
& \frac{\partial \theta}{\partial t}-\theta^{\prime \prime}-u \theta^{\prime}+k^{2} \theta=\left\{\begin{array}{lr}
u \exp \left(-u z_{2}\right) v, & z_{2}>\xi, \\
0, & z_{2}<\xi,
\end{array}\right.
\end{aligned}
$$

with the above jump conditions (4.6)-(4.7). The problem (4.9)-(4.11) subject to the jump conditions (4.6)-(4.7) is an eigenvalue problem with time-dependencies coefficients. We solve it numerically by using the finite-difference approximation with implicit scheme except for the boundary condition where the velocity $v$ is taken from the previous time step (see [1]), and find the stability boundary.

\section{Numerical results and discussion}

Figure 1 shows the profiles of the perturbed temperature and velocity for $R=45, Z=8, k=$ 1.785, $P=0.5$ and $\lambda=0$ which means that there is no influence of vibrations, we obtain the same result as in [3] (considered as a limiting model of the present one when $\lambda \rightarrow 0$ ). For fixed $Z$ and $P$ we vary $R$. If the Rayleigh number $R$ is less than a critical value $R_{c r}$, then the solution decreasing in time (Figure 2). If $R>R_{c r}$, it increases, and for $R=R_{c r}$ it is periodic in time. Figure 3 shows the critical value of the Rayleigh number as a function of the amplitude of vibrations for different frequencies. If $\lambda=0$, we obtain the same value $\left(R_{c r} \approx 27, k=0.7\right)$ as for the case without vibrations [3]. For small positive $\lambda$ vibrations stabilize the solution: $R_{c r}$ is an increasing function. For larger $\lambda$ there appears a decreasing branch of the stability boundary. It corresponds to a parametric instability where vibrations with high amplitude destabilize the front. In Figure 4 we draw the critical Rayleigh number as a function of wave number for $Z=8, P=0.5, \lambda=0$ (solid line, without vibrations) $\lambda=5$ (dotted line with vibrations) and $\nu=10$. This shows that the vibrations stabilize the ascending fronts.

In this work we have studied the influence of vibrations on the convective instability of a polymerization front with a liquid monomer and a liquid polymer. We have shown that vibrations can change the onset of convection. We note finally that the case where the polymer is liquid is essentially different from the case of a solid polymer considered in [1,2]. The most essential difference is that the convective instability exists for descending fronts also (see [3]). The influence of vibrations on the descending fronts is under study as a continuation of this work.

\section{References}

[1] K. Allali, J. Pojman, V. Volpert. Influence of vibrations on convective instability of polymerization fronts. J. of Engineering Mathematics, 41 (2001), 13-31. 


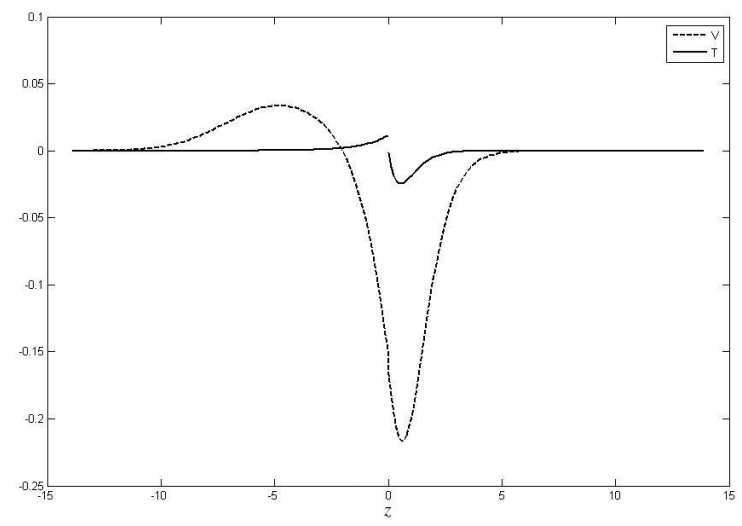

Figure 1: Velocity (dashed line) and temperature (solid line) distributions in the case without vibrations for $R=45, k=1.785, Z=8$, $P=0.5$ and $\lambda=0$ (no vibration).

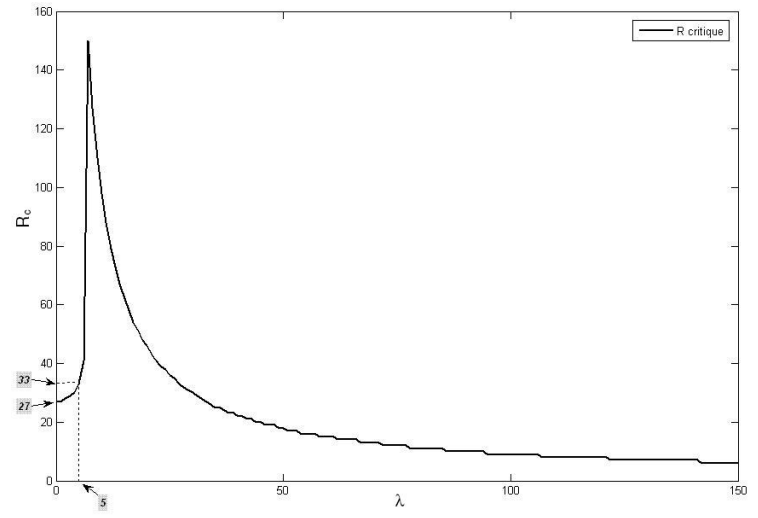

Figure 3: Convective instability boundary: critical Rayleigh number as a function of the amplitude of vibrations for $k=0.7, Z=8$, $P=0.5$ and $\mu=10$.

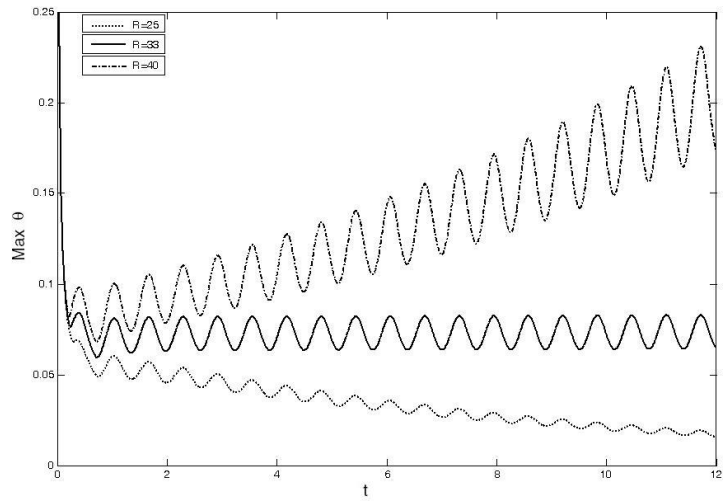

Figure 2: Maximum temperature with vibration for different value of $R$ with $k=0.7$, $Z=8, P=0.5, \lambda=5$ and $\mu=10$.

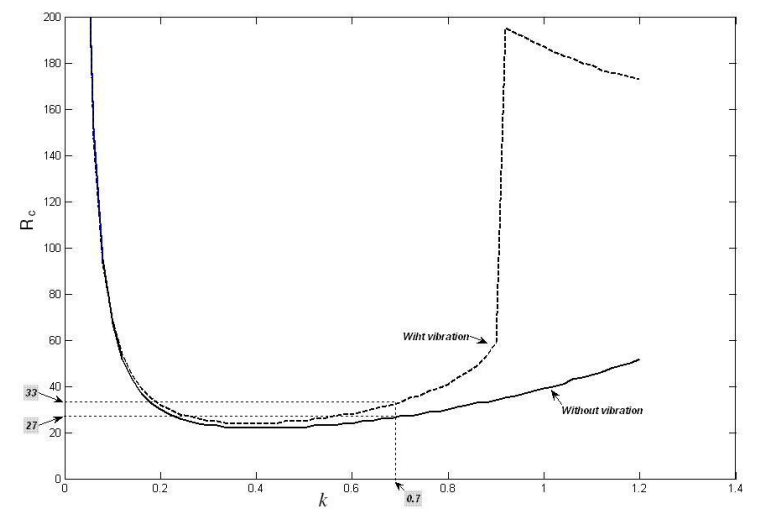

Figure 4: Convective instability boundary: critical Rayleigh number as a function of wave number for $Z=8, P=0.5, \lambda=5$ and $\mu=10$.

[2] M. Garbey, A. Taik, V. Volpert. Linear stability analysis of reaction fronts in liquids. Quart. Appl. Math., 1996, No. 54, 225-247.

[3] M. Garbey, A. Taik, V. Volpert. Influence of natural convection on stability of reaction fronts in liquids. Quart. Appl. Math., 1998, No. 53, 1-35.

[4] B.J. Matkowsky, G.I. Sivashinsky. An asymptotic derivation of two models in flame theory associated with the constant density approximation. SIAMJ. Appl. Math., 37 (1979), 686699. 
[5] B.V. Novozhilov. The rate of propagation of the front of an exothermic reaction in a condensed phase. Proc. Acad. Sci. URSS , Phys. Chem. Sect., 141 (1961), 836-838.

[6] Ya.B. Zeldovich, G.I. Barenblatt, V.B. Librovich, G.M. Makhviladze. The mathematical theory of combustion and explosions. New York: Consultants Bureau (1985).

[7] Ya.B. Zeldovich, D.A. Frank-Kamenetskii. Theory of thermal propagation of flames, Zh. Fiz. Khim., 12, 100, 1938. 